\title{
Kinetic Modeling of 3'-Deoxy-3'-18F- Fluorothymidine for Quantitative Cell Proliferation Imaging in Subcutaneous Tumor Models in Mice
}

\author{
Su Jin Kim ${ }^{1-4}$, Jae Sung Lee ${ }^{1-4}$, Ki Chum Im ${ }^{5,6}$, Seog-Young Kim ${ }^{5,6}$, Soo-Ah Park ${ }^{5,6}$, Seung Jin Lee ${ }^{5,6}$, \\ Seung Jun $\mathrm{Oh}^{5,6}$, Dong Soo Lee ${ }^{1-4}$, and Dae Hyuk Moon ${ }^{5,6}$ \\ ${ }^{1}$ Department of Nuclear Medicine, College of Medicine, Seoul National University, Seoul, Korea; ${ }^{2}$ Department of Biomedical Sciences, \\ College of Medicine, Seoul National University, Seoul, Korea; ${ }^{3}$ Interdisciplinary Programs in Radiation Applied Life Science, College of \\ Medicine, Seoul National University, Seoul, Korea; ${ }^{4}$ Institute of Radiation Medicine, Medical Research Center, Seoul National \\ University, Seoul, Korea; ${ }^{5}$ Department of Nuclear Medicine, Asan Medical Center, University of Ulsan College of Medicine, Seoul, \\ Korea; and ${ }^{6}$ Institute for Innovative Cancer Research, Asan Medical Center, Seoul, Korea
}

$3^{\prime}$-Deoxy-3'-18 F-fluorothymidine $\left({ }^{18} \mathrm{~F}-\mathrm{FLT}\right)$ is a thymidine analog that was developed for measuring tumor proliferation with PET. The aim of this study was to establish a kinetic modeling analysis method for quantitative ${ }^{18} \mathrm{~F}-\mathrm{FLT}$ PET studies in subcutaneous tumor models in mice. Methods: To explore the validity of an image-derived left ventricular input function, we measured equilibrium constants for plasma and whole blood and metabolite fractions in blood after ${ }^{18} \mathrm{~F}-\mathrm{FLT}$ injection. In parallel, dynamic ${ }^{18} \mathrm{~F}$-FLT PET scans were acquired in 24 mice with a small-animal dedicated PET scanner to compare arterial blood activities obtained by PET and blood sampling. We then investigated kinetic models for ${ }^{18} \mathrm{~F}-\mathrm{FLT}$ in human epithelial carcinoma (A431) and Lewis lung carcinoma tumor models in mice. Three-compartment models with reversible phosphorylation $\left(k_{4} \neq 0,3 \mathrm{C} 5 \mathrm{P}\right)$ and irreversible phosphorylation $\left(k_{4}=0,3 \mathrm{C} 4 \mathrm{P}\right)$ and a 2-compartment model (2C3P) were examined. The Akaike information criterion and $F$ statistics were used to select the best model for the dataset. Gjedde-Patlak graphic analysis was performed, and standardized uptake values in the last frame were calculated for comparison purposes. In addition, quantitative PET parameters were compared with $\mathrm{Ki}-67$ immunostaining results. Results: ${ }^{18} \mathrm{~F}-\mathrm{FLT}$ equilibrated rapidly (within $30 \mathrm{~s}$ ) between plasma and whole blood, and metabolite fractions were negligible during PET scans. A high correlation between arterial blood sampling and PET data was observed. For 120-min dynamic PET data, the 3C5P model best described tissue time-activity curves for tumor regions. The net influx of ${ }^{18} \mathrm{~F}-\mathrm{FLT}\left(K_{\mathrm{FLT}}\right)$ and $k_{3}$ obtained with this model showed reasonable intersubject variability and discrimination ability for tumor models with different proliferation properties. The $K_{\mathrm{FLT}}$ obtained from the 60- or 90-min data correlated well with that obtained from the 120-min data as well as with the Ki-67 results. Conclusion: The image-derived arterial in-

Received Apr. 8, 2008; revision accepted Aug. 18, 2008.

For correspondence or reprints contact: Jae Sung Lee, Departments of Nuclear Medicine and Biomedical Sciences, College of Medicine, Seoul National University, 28 Yungun-Dong, Chongno-Gu, Seoul 110-744, Korea. E-mail: jaes@snu.ac.kr

COPYRIGHT @ 2008 by the Society of Nuclear Medicine, Inc. put function was found to be feasible for kinetic modeling studies of ${ }^{18} \mathrm{~F}$-FLT PET in mice, and kinetic modeling analysis with an adequate compartment model provided reliable kinetic parameters for measuring tumor proliferation.

Key Words: $3^{\prime}$-deoxy-3'-18 $\mathrm{F}$-fluorothymidine $\left({ }^{18} \mathrm{~F}-\mathrm{FLT}\right)$; cellular proliferation; kinetic modeling; arterial input function

J Nucl Med 2008; 49:2057-2066

DOI: 10.2967/jnumed.108.053215

$\mathbf{T}$ he thymidine analog $3^{\prime}$-deoxy- $3^{\prime}-{ }^{18}$ F-fluorothymidine $\left({ }^{18} \mathrm{~F}-\mathrm{FLT}\right)$ is used to assess rate-controlling enzyme thymidine kinase 1 (TK1) activity in the DNA salvage pathway and hence cellular proliferation $(1,2)$. Compared with ${ }^{11} \mathrm{C}$ thymidine, ${ }^{18} \mathrm{~F}$-FLT offers the advantages of a longer physical half-life and the generation of few metabolites in vivo. Furthermore, because it is not incorporated into DNA, ${ }^{18} \mathrm{~F}-\mathrm{FLT}$ is trapped in cells like ${ }^{18} \mathrm{~F}-\mathrm{FDG}(3)$.

For quantifying ${ }^{18} \mathrm{~F}$-FLT uptake in vivo, simple semiquantitative methods, such as determination of the standardized uptake value (SUV) and model-independent graphic analysis, have been shown to be useful for assessing ${ }^{18}$ F-FLT metabolism (4-7). However, compartmental modeling is necessary to fully characterize the kinetics of ${ }^{18} \mathrm{~F}$-FLT uptake into tissue (8). Several investigations of ${ }^{18} \mathrm{~F}$-FLT kinetic modeling in humans have been performed $(6,8-13)$. In vivo imaging studies of experimental cancer models in mice have provided important means of understanding the mechanisms of cancer progression and of assessing the therapeutic effects of newly developed drugs (14-17). Serial ${ }^{18}$ F-FLT PET studies of the monitoring of tumor response to radiotherapy or antiproliferative treatment $(3,7,18-20)$ in various tumor models have been performed. However, only semiquantitative approaches were used to assess the relationship between 
changes in ${ }^{18} \mathrm{~F}$-FLT uptake and therapeutic responses in these studies.

In mice, left ventricular (LV) activity can be used as an image-derived input function for quantifying ${ }^{18} \mathrm{~F}$-FLT metabolism because no ${ }^{18} \mathrm{~F}$-FLT metabolites are found in plasma, but the data so derived need to be validated. Therefore, in this study, we explored the validity of an image-derived LV input function in mouse ${ }^{18} \mathrm{~F}$-FLT PET and investigated kinetic models for ${ }^{18} \mathrm{~F}-\mathrm{FLT}$ in mice bearing human epithelial carcinoma (A431) and Lewis lung carcinoma (LLC) tumors.

\section{MATERIALS AND METHODS}

\section{Radiopharmaceutical Preparation}

${ }^{18} \mathrm{~F}$-FLT was prepared from $5^{\prime}-O$-(DMTr-2'-deoxy-3'-O-nosyl$\beta$-D-threopentafuranosyl)-3- $N$-BOC-thymine as a precursor by the nucleophilic fluorination of ${ }^{18} \mathrm{~F}$-fluoride with a protic solvent ( $t$-butanol or $t$-amyl alcohol) (21). Typically, decay-corrected radiochemical yields ranged from $60 \%$ to $70 \%$, and after highperformance liquid chromatography (HPLC) purification, the radiochemical purity was $98 \% \pm 1.2 \%$ (mean $\pm \mathrm{SD}$ ). The specific activity of the ${ }^{18} \mathrm{~F}$-FLT obtained was greater than $100 \mathrm{TBq} / \mathrm{mmol}$.

\section{Cell Cultures and Tumor Models}

A431 and LLC cell lines were obtained from the American Type Culture Collection. Cells were routinely cultured in Dulbecco's modified Eagle medium supplemented with 10\% heat-inactivated fetal bovine serum (FBS), L-glutamine $(2 \mathrm{mM})$, penicillin (100 IU/ $\mathrm{mL}$ ), and streptomycin ( $50 \mathrm{~g} / \mathrm{mL})$ (Gibco, Invitrogen Corp.). Cells were maintained at $37^{\circ} \mathrm{C}$ in an atmosphere of $5 \% \mathrm{CO}_{2}$ in air.

BALB-c/nu and C57BL/6 mice (both from Charles River Laboratories) subcutaneously injected with A431 and LLC cells, respectively, were used as tumor models. The research protocol used was approved by the Institutional Animal Care and Use Committee at the Asan Institute for Life Science. Mice were also maintained in accordance with guidelines issued by this committee. Exponentially growing $7 \times 10^{6} \mathrm{~A} 431$ and $1 \times 10^{6} \mathrm{LLC}$ cells suspended in $300 \mu \mathrm{L}$ of the culture medium described earlier were injected subcutaneously into the right forelimbs of anesthetized mice. When tumor diameters reached 6-10 $\mathrm{mm}$ at $10-12 \mathrm{~d}$ after injection, mice were used for the experiments.

\section{Thymidine Kinase Assay}

Thymidine kinase activities were measured in A431 and LLC tumors excised from 3 and 4 mice, respectively, that were treated in parallel with animals used for imaging. The activities were measured with a previously described thymidine kinase assay that was slightly modified (1). In brief, tumors were lysed in lysis buffer and incubated for $30 \mathrm{~min}$ on ice. Lysates were centrifuged at $10,000 \mathrm{~g}$ for $20 \mathrm{~min}$ at $4^{\circ} \mathrm{C}$, and supernatants were recentrifuged at $100,000 \mathrm{~g}$ for $1 \mathrm{~h}$ at $4^{\circ} \mathrm{C}$ to separate mitochondrial fractions. After protein contents were determined, cytosolic fractions were assayed for thymidine kinase activities in a reaction buffer containing $10 \mathrm{M}^{3} \mathrm{H}$-thymidine (TRK120; $925 \times 10^{9} \mathrm{~Bq} / \mathrm{mmol}$; Amersham Bioscience). Mixtures were then incubated at $37^{\circ} \mathrm{C}$ with gentle stirring, and samples were removed and added to $10 \mathrm{mM}$ ethylenediaminetetraacetic acid to stop the reaction. For sequestration of labeled nucleotides, samples were spotted onto DE-81 filters (Whatman), dried, and washed in $4 \mathrm{mM}$ ammonium formate and $95 \%$ ethanol. Radioactivity was measured by liquid scintillation counting with Ultima Gold $\mathrm{F}$ scintillation cocktail solution (Perkin-Elmer). Activities were cal- culated with linear time-activity curves and are presented as picomoles of phosphorylated thymidine per minute per milligram of protein. TK1 activities (mean \pm SD) in A431 and LLC tumors were $0.074 \pm 0.023$ and $0.028 \pm 0.01$ pmol of phosphorylated thymidine per minute per milligram of protein, respectively; these values were significantly different $(P<0.05)$.

\section{PET Scans}

PET scans were performed by use of a microPET Focus 120 system (Siemens Medical Solutions, Inc.) with resolutions of 1.18 $\mathrm{mm}$ (radial), $1.13 \mathrm{~mm}$ (tangential), and $1.44 \mathrm{~mm}$ (axial) at the center of the field of view (22). A 37-frame dynamic protocol $(4 \times 3 \mathrm{~s}, 6 \times$ $1 \mathrm{~s}, 7 \times 6 \mathrm{~s}, 8 \times 30 \mathrm{~s}, 1 \times 300 \mathrm{~s}$, and $11 \times 600 \mathrm{~s}$ ) was used for the emission PET scans. Mice were maintained under isoflurane anesthesia during the scans, and body temperatures were maintained at $36^{\circ} \mathrm{C}$ with an electric heating pad. Animals fasted for $4 \mathrm{~h}$ before imaging. Transaxial images were reconstructed as $128 \times 128 \times 95$ matrices of $0.432 \times 0.432 \times 0.796 \mathrm{~mm}$ by use of a filtered backprojection algorithm with a Hamming filter at a cutoff frequency of 0.5 cycle per pixel.

\section{Validation of LV Input Function}

Forty-four normal C57BL/6 mice were studied to verify the LV input function derived from dynamic PET data. For measurement of the equilibrium constant for plasma and whole blood over time, arterial blood samples were obtained from 16 mice by cardiac puncture at $30 \mathrm{~s}, 2 \mathrm{~min}, 5 \mathrm{~min}$, and $10 \mathrm{~min}(4 \mathrm{mice}$ at each sampling time point) after injection of $37 \mathrm{MBq}$ of ${ }^{18} \mathrm{~F}$-FLT. Arterial blood was collected in heparinized tubes from the LV cavity by direct cardiac puncture. Immediately after sample collection, plasma was separated from whole blood by centrifugation at $10,000 \mathrm{~g}$ for $20 \mathrm{~s}$ at $4^{\circ} \mathrm{C}$. The plasma samples $(>15 \mu \mathrm{g})$ were weighed with $0.1-\mathrm{mg}$ precision. Counts in the plasma and whole-blood samples were determined with a well $\gamma$-counter (COBRA II Auto Gamma; Canberra Packard). All tubes were incubated at $4^{\circ} \mathrm{C}$ before use. The time interval between plasma sampling after centrifugation and the sacrifice of the animal was $53 \pm 11 \mathrm{~s}$.

Dynamic PET scans were performed in 24 mice to compare arterial blood activities obtained by PET with activities obtained from blood data. The 24 animals were divided into 4 equal groups according to the time of arterial blood sampling $(5,10,30$, and 60 min after the initiation of scanning). Dynamic PET scans were started immediately after the injection of $37 \mathrm{MBq}$ of ${ }^{18} \mathrm{~F}$-FLT into tail veins and were completed at each group time for blood sampling by direct cardiac puncture. Arterial blood samples $(20 \mu \mathrm{l})$ were analyzed with the well $\gamma$-counter.

Cross-calibration factors for the well $\gamma$-counter and PET scanner were independently measured with a mouse-size uniform cylindric phantom (diameter $=3 \mathrm{~cm}$, length $=11.5 \mathrm{~cm}$ ) filled with $37 \mathrm{MBq}$ of ${ }^{18}$ F-FLT solution. To obtain a PET image-derived input function, cylindric volumes of interest (VOIs) (length of 3 slices) were drawn on the centers of the left ventricles on PET images. The diameters of these cylindric VOIs were varied from 1.3 to $3.0 \mathrm{~mm}$ (3-7 pixels in diameter) to explore the effects of VOI size on the image-derived input function. Finally, correlation analysis was performed on crosscalibrated counts from arterial blood and image-derived input functions. The ratios of cross-calibrated PET image-derived and blood sample-derived activities (recovery coefficients) were also calculated, and the reciprocal of the mean ratio of the data for the 24 imaged animals (at all time points between 5 and $60 \mathrm{~min}$ ) was used 
as a partial-volume effect correction factor for the image-derived input function.

\section{${ }^{18}$ F-FLT Metabolite Analysis}

Metabolite fractions in arterial blood were measured at 1 and $2 \mathrm{~h}$ after the injection of $185 \mathrm{MBq}$ of ${ }^{18} \mathrm{~F}$-FLT into 4 mice as described previously (3). Plasma samples were deproteinated by the addition of ice-cold acetonitrile and centrifugation $(1,800 \mathrm{~g}, 10 \mathrm{~min}) . \mathrm{Su}-$ pernatants containing ${ }^{18} \mathrm{~F}-\mathrm{FLT}$ and metabolites were passed through a sterile filter $(0.2 \mu \mathrm{m})$ and analyzed by HPLC. Eluted material was monitored with a radioactivity detector, and unchanged phosphorylated ${ }^{18} \mathrm{~F}$-FLT fractions were determined by HPLC with radioisotope detectors.

Metabolite fractions in tumors were also measured immediately after dynamic imaging studies as described later. Tumor samples were cut into small pieces and homogenized. Supernatants obtained by centrifugation were filtered and analyzed as described earlier.

\section{Kinetic Modeling}

For kinetic modeling studies, PET scans were performed on 19 mice bearing A431 $(n=9)$ and LLC $(n=10)$ tumors. Dynamic emission scans were acquired after the injection of $30-46 \mathrm{MBq}$ of ${ }^{18} \mathrm{~F}-\mathrm{FLT}$ into tail veins, and scanning was continued for $2 \mathrm{~h}$.

To obtain time-activity curves for kinetic analysis, cylindric VOIs with a diameter of $3.0 \mathrm{~mm}$ and a length of 3 slices were drawn on PET images. LV time-activity curves corrected for partialvolume effects with the correction factor described earlier were used as the input function. Tissue time-activity curves were obtained from tumor-bearing regions on the right forelimbs and from normal contralateral regions.

\section{Compartmental Analysis}

To determine the optimal compartmental model for ${ }^{18} \mathrm{~F}$-FLT in mice, we tested a 3-compartment model suggested for human data $(8,9)$ and its nested models. Figure 1 shows the 3-compartment model, in which the parameters $K_{1}, k_{2}, k_{3}$, and $k_{4}$ represent the rate of transport from plasma to tissue, the rate of outflow from tissue to plasma, the TK1 phosphorylation rate, and the dephosphorylation rate, respectively. The blood volume fraction $\left(V_{\mathrm{b}}\right)$ was included in the modeling, and 3-compartment models with reversible phosphorylation $\left(k_{4} \neq 0,3 \mathrm{C} 5 \mathrm{P}\right)$ and irreversible phosphorylation $\left(k_{4}=0\right.$, $3 \mathrm{C} 4 \mathrm{P})$ were examined. In addition, a 2-compartment model that combined exchangeable and phosphorylated compartments into a single tissue compartment with 3 parameters (2C3P: $K_{1}, k_{2}$, and $V_{\mathrm{b}}$ ) was also considered.

The PMOD software package (version 2.65; PMOD Group (23)) was used for parameter estimation. Tissue time-activity curves

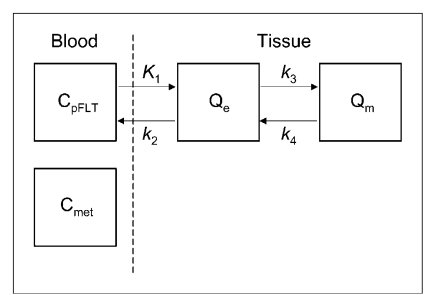

FIGURE 1. Kinetic model for ${ }^{18} \mathrm{~F}-\mathrm{FLT}$. $\mathrm{C}_{\mathrm{pFLT}}$ represents concentration of ${ }^{18} \mathrm{~F}-\mathrm{FLT}$ in arterial plasma. Tissue is composed of exchangeable tissue compartment $\left(Q_{e}\right)$ and compartment of trapped phosphorylated ${ }^{18} \mathrm{~F}-\mathrm{FLT}$ nucleotides $\left(\mathrm{Q}_{\mathrm{m}}\right)$. Parameters $K_{1}$ and $k_{2}$ are rate constants for forward and reverse transport of ${ }^{18} \mathrm{~F}-\mathrm{FLT}$ between plasma and tissue pool, respectively. Rate constants for precursor and phosphorylated ${ }^{18} \mathrm{~F}-\mathrm{FLT}$ denote thymidine kinase-mediated phosphorylation $\left(k_{3}\right)$ and dephosphorylation $\left(k_{4}\right)$ of ${ }^{18} \mathrm{~F}-\mathrm{FLT}$. $\mathrm{C}_{\mathrm{met}}=$ concentration of metabolites in arterial plasma. were fitted to the models by use of the nonlinear least-squares method with the Levenberg-Marquardt algorithm, which minimizes the weighted sum of squared errors between PET measurements and model solutions. Inverse SDs of frame counts were used as weights. Estimated parameters were restricted to the following value ranges: $0.0-0.5$ for $K_{1}, 0.0-5.0$ for $K_{1} / k_{2}, 0.0-1.0$ for $k_{3}, 0.0$ 0.5 for $k_{4}$, and $0.0-0.2$ for $V_{\mathrm{b}}$. Initial values for these parameters were set at $0.1,1.0,0.1,0.01$, and 0.05 for A431 and at 0.1, 0.5, $0.01,0.01$, and 0.05 for LLC, respectively.

The net influx of ${ }^{18} \mathrm{~F}-\mathrm{FLT}\left(K_{\mathrm{FLT}}\right)$, the total distribution volume $\left(D V_{\text {tot }}\right)$, and the distribution volume for phosphorylated ${ }^{18} \mathrm{~F}-\mathrm{FLT}$ nucleotides $\left(D V_{\mathrm{m}}\right)$ were estimated as follows:

$$
K_{F L T}=\frac{K_{1} k_{3}}{k_{2}+k_{3} \ldots} D V_{t o t}=\frac{K_{1}}{k_{2}}\left(1+\frac{k_{3}}{k_{4}}\right)_{\ldots} D V_{m}=\frac{K_{1} k_{3}}{k_{2} k_{4}} .
$$

We chose an adequate compartment model on the basis of the Akaike information criterion (AIC) and the $F$ test. We also examined the correlations between kinetic parameters (such as $K_{\mathrm{FLT}}$, $D V_{\text {tot }}$, and $D V_{\mathrm{m}}$ ) obtained with the best model and percentages of Ki-67-positive nuclei (\% Ki-67). Kinetic parameters obtained with simpler methods (fewer parameters or a shorter scan duration) were compared with those obtained with the best model and data acquired over $120 \mathrm{~min}$.

\section{Nonparametric or Semiquantitative Approaches}

Gjedde-Patlak graphic analysis (GPGA) was also performed to estimate $K_{\text {FLT }}$. Only the linear region on the GPGA plot (A431: 7.5-90 $\mathrm{min}$; LLC: $20-120 \mathrm{~min}$ ) was included in the linear regression analysis. The SUV at the last frame (duration of $10 \mathrm{~min}$ ) was also calculated.

\section{Immunohistochemical Analysis}

After small-animal PET images were acquired from mice bearing tumors, animals were sacrificed for immunohistochemical analysis. Tumors were fixed with $10 \%$ neutral buffered formalin and embedded in paraffin. For Ki-67 immunostaining, 4- $\mu$ m-thick sections were obtained from paraffin blocks. Paraffin sections were placed on slides, deparaffinized in xylene, and rehydrated in graded ethanol. Endogenous peroxidase was blocked with $3 \%$ hydrogen peroxide in $70 \%$ methanol for $15 \mathrm{~min}$. Antigen retrieval was performed with 10 $\mathrm{mM}$ citrate buffer solution ( $\mathrm{pH}$ 6.0) for $15 \mathrm{~min}$ in a microwave oven, and sections were cooled to room temperature for $20 \mathrm{~min}$. Sections were incubated overnight at $4^{\circ} \mathrm{C}$ with monoclonal mouse antihuman Ki-67 antibody. Sections were treated with biotinylated Link (LSAB 2 system-HRP kit; Dakocytomation) for $30 \mathrm{~min}$, incubated with streptavidin-horseradish peroxidase (LSAB 2 system-HRP kit) for $30 \mathrm{~min}$ at room temperature, and washed. Sections were treated with diaminobenzidine (Dakocytomation) for $5 \mathrm{~min}$, washed with tap water, counterstained with hematoxylin, rewashed, and mounted. For determination of $\% \mathrm{Ki}-67$, more than 1,000 cells per slide were counted and scored with a $\times 40$ optical microscope (Leica) by 2 masked observers.

\section{Statistical Analysis}

Correlations between $\% \mathrm{Ki}-67$ and parameters estimated from PET data were assessed with Spearman nonparametric rank analysis. Correlations between PET kinetic parameters were also assessed with Spearman analysis. The 2-sample $t$ test was used to compare 2-parameter estimates from different tumors. Statistical analyses were performed with SPSS for Windows (SPSS 12.0KO release 12.0.1; SPSS Inc.). 


\section{Image-Derived Input Function}

${ }^{18} \mathrm{~F}-\mathrm{FLT}$ equilibrated rapidly between plasma and whole blood, and the ratios of plasma activity to whole-blood activity converged to a constant value (mean \pm SD for 30-s data $=1.0 \pm 0.1$ ) within $30 \mathrm{~s}$ of intravenous injection and did not change with time. Metabolite analysis in plasma revealed only one major peak of parent ${ }^{18} \mathrm{~F}-\mathrm{FLT}$, at $2 \mathrm{~h}$ after injection. Figure 2 shows the LV input function obtained for a representative mouse with a VOI with a diameter of $1.3 \mathrm{~mm}$ (3 pixels), which was the smallest VOI used. We obtained several samples around the initial peaks of LV input functions. Despite the short duration (1 s) of the initial frames, no counting rate fluctuation was observed for these input functions throughout the scans. Initial peak values of imagederived input functions decreased gradually with increasing VOI diameter. However, these differences were not significant up to a VOI diameter of $3 \mathrm{~mm}$ (7 pixels).

Figure 3A shows the regression line for the crosscalibrated arterial blood sample data and PET data (timing difference corrected) obtained with a VOI with a $3-\mathrm{mm}$ diameter. We merged the data acquired at all of the time points $(5,10,30$, and $60 \mathrm{~min})$ to obtain the regression line, which had a high correlation $(r>0.96)$ and little bias. Regression lines for all VOIs showed a similar trend. The magnitudes of arterial blood sample data and PET data obtained at each time point with the same VOIs are compared in Figure 3B, in which the data are presented as means \pm SEMs. No time-dependent difference between these data was observed.

Figure 3C shows recovery coefficients, which were defined as ratios of PET data and blood sample data (mean \pm

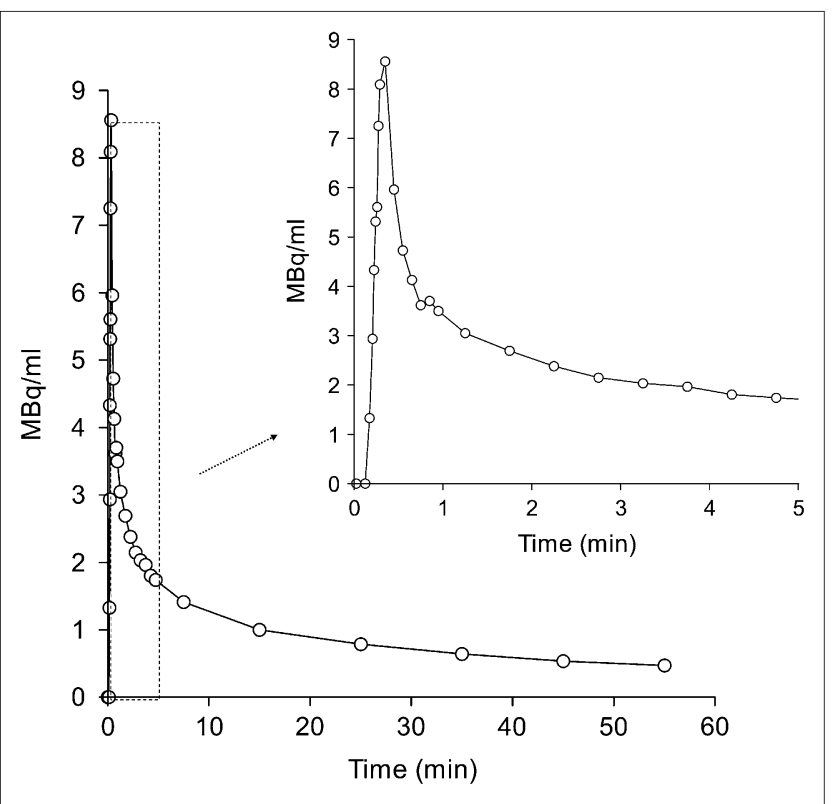

FIGURE 2. Blood time-activity curve generated from VOI (1.3$\mathrm{mm}$ diameter) placed in left ventricle to obtain input function.
FIGURE 3. Comparison between cross-calibrated arterial blood activities measured by invasive blood sampling and by use of LV VOls on PET images. (A) High correlation $(r>0.96)$ was observed between arterial blood samples and PET data (VOI diameter $=3$ $\mathrm{mm})$. (B) Bar plot of arterial blood samples and PET data obtained from each time point with same VOI. Bars represent means \pm SEMs. (C) Mean ratios of PET data and blood sample data were 0.94, 0.94, 0.93, 0.93 , and 0.92 for VOls with diameters of 1.3, 1.7, $2.1,2.6$, and $3 \mathrm{~mm}$, respectively. Input function obtained from VOI with diameter of $3 \mathrm{~mm} \mathrm{(7}$ pixels) was divided by 0.92 for kinetic analysis. Bars represent means \pm SEMs.
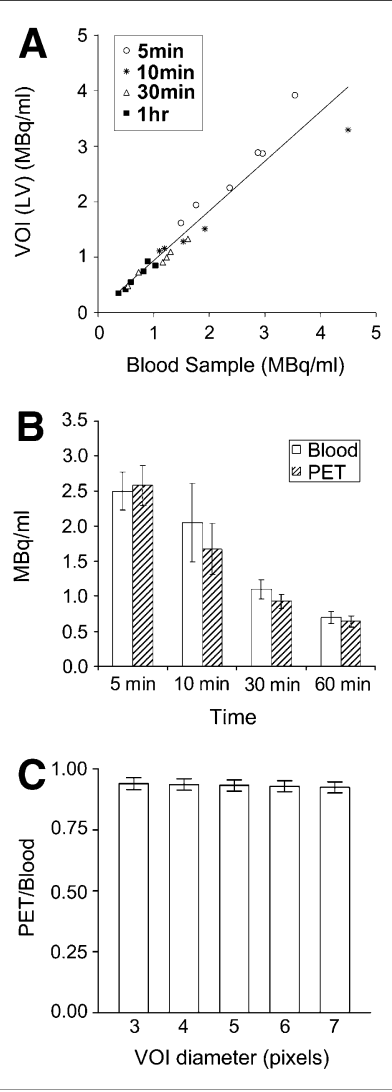

SD of $0.94 \pm 0.12,0.94 \pm 0.11,0.93 \pm 0.11,0.93 \pm 0.10$ and $0.92 \pm 0.11$ for VOIs with diameters of 1.3, 1.7, 2.1, 2.6, and $3 \mathrm{~mm}$, respectively). Coefficients of variation (CVs) were less than $12.5 \%$.

On the basis of these results, the LV time-activity curves obtained with a VOI with a 3-mm diameter (7 pixels) were used as input functions for the kinetic analysis. Input functions were scaled to compensate for differences from blood samples by dividing them by recovery coefficients.

\section{Tissue Time-Activity Curves}

Figure 4 shows averaged PET images obtained at 110-120 min after injection and time-activity curves for tumor-bearing and normal regions. Proliferative A431 tumors showed rapid uptake and high ${ }^{18} \mathrm{~F}$-FLT retention (Fig. 4A). On the other hand, LLC tumors had time-activity curves that resembled those of normal regions and had relatively low ${ }^{18}$ F-FLT retention and rapid clearance (Fig. 4B).

\section{Compartmental Modeling}

Time-activity curves were fitted more accurately when the $V_{\mathrm{b}}$ term, which reflected the significant amount of blood activity in VOIs, was included in the model. Means and CVs of kinetic parameters estimated with the 3C5P, 3C4P, and $2 \mathrm{C} 3 \mathrm{P}$ models and $120 \mathrm{~min}$ of data and $K_{\mathrm{FLT}}$ values estimated with GPGA are summarized in Table 1. Data for 2 A431 tumors were excluded from this comparison and from further analyses because tissue time-activity curves 


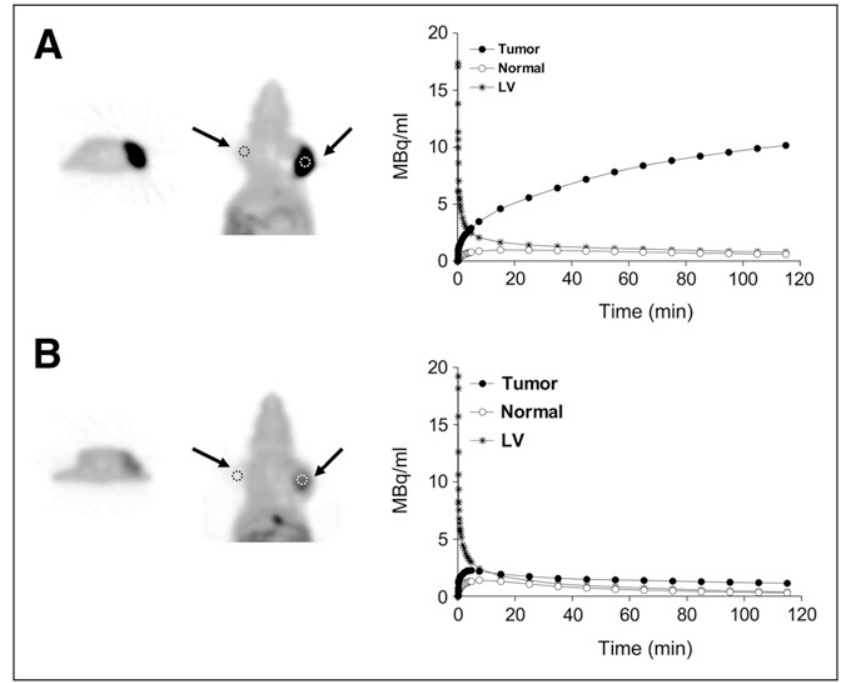

FIGURE 4. Averaged PET images obtained at $110-120 \mathrm{~min}$ after injection (representative case for each tumor model). LV input function and tissue time-activity curves were obtained from 3-mm VOls drawn on tumor-bearing regions (arrows at right) and on contralateral normal tissues (arrows at left) in mice bearing $A 431(A)$ and LLC (B) tumors.

were not properly fitted with the models within physiologically relevant ranges of kinetic parameters, possibly because of motion artifacts. For A431 and LLC tumors, the average ${ }^{18} \mathrm{~F}$-FLT dephosphorylation rates $\left(k_{4}\right)$ were estimated to be 0.011 and 0.015 , respectively.

Consistent with the measurable dephosphorylation of phosphorylated ${ }^{18} \mathrm{~F}$-FLT, both tumor cell lines showed the lowest AIC values for all mice when the 3C5P model was used for curve fitting (Table 2). The results of $F$ tests were similar to those of AIC analysis (Table 2). AIC values from the 3C models (3C5P and 3C4P) were lower than those from the $2 \mathrm{C} 3 \mathrm{P}$ model in all mice, and differences were significant, except for one mouse with A431 $(P<0.001)$. The quality of curve fitting with different models was evaluated, and the results are shown in Figure 5.
Correlation matrices for the $3 \mathrm{C} 5 \mathrm{P}$ model are shown in Table $3 . K_{1} / k_{2}$ and $k_{3}$ were found to be highly correlated with each other in both tumor cell lines. In LLC, $k_{3}$ and $k_{4}$ were highly correlated. Because a high level of covariance between microparameters indicates difficulties in independent estimation, $K_{\mathrm{FLT}}$ values obtained from the different compartment models were further analyzed. As was expected, $K_{\mathrm{FLT}}$ values estimated with 3C5P were much higher than those estimated with $3 \mathrm{C} 4 \mathrm{P}$ or GPGA (Table 1). On average, $K_{\mathrm{FLT}}$ values were underestimated by approximately $33 \%$ (range, $-48 \%$ to $-17 \%$ ) for A431 tumors and by $56 \%$ (range, $-69 \%$ to $-39 \%$ ) for LLC tumors when $k_{4}$ was set to 0 (3C4P vs. $3 \mathrm{C} 5 \mathrm{P}$ ). $K_{\mathrm{FLT}}$ values estimated with GPGA were also consistently lower than those estimated with the 3C5P model (for A431: mean $=-26 \%$ and range $=-38 \%$ to $-11 \%$; for LLC: mean $=-67 \%$ and range $=-80 \%$ to $-53 \%) . K_{\mathrm{FLT}}$ composed of microparameters $\left(K_{1} \sim k_{3}\right)$ was found to be better correlated with $k_{3}$ than $K_{1}$, as shown in Figure 6.

\section{Correlation of Model Parameters with $\% \mathrm{Ki}-67$ and ${ }^{18} \mathrm{~F}-$ FLT Phosphorylation}

A431 and LLC tumors showed markedly different levels of quantitative and semiquantitative parameters related to ${ }^{18}$ F-FLT metabolism $\left(k_{3}, K_{\mathrm{FLT}}\right.$, and $\left.D V_{\mathrm{m}}\right)(P<0.01)$ (Table 1). In addition, significant differences in SUVs were noted between the 2 tumor types $(6.95 \pm 1.14$ for A431 and $0.13 \pm$ 0.04 for LLC) and $\% \mathrm{Ki}-67$ (81.5 \pm 4.4 for A431 and $58.7 \pm$ 6.3 for LLC) $(P<0.01)$.

The radiochromatograms of A431 tumors obtained $2 \mathrm{~h}$ after ${ }^{18} \mathrm{~F}$-FLT injection showed that the major radioactive component, which corresponded to phosphorylated ${ }^{18} \mathrm{~F}-\mathrm{FLT}$, eluted at a retention time of 2-3 min. No other radioactive peaks were identified. On the other hand, the radiochromatograms of LLC tumors did not contain well-identified peaks. In some tumors, a major peak corresponding to phosphorylated ${ }^{18} \mathrm{~F}-\mathrm{FLT}$ and a minor peak corresponding to intact ${ }^{18} \mathrm{~F}-$ FLT were identified at retention times of 9-10 min. Although fractions of these 2 components were not measured, the results of radio-HPLC were in qualitative agreement with the

TABLE 1

Kinetic Parameters for ${ }^{18}$ F-FLT PET in Mice Implanted with A431 and LLC Tumors

\begin{tabular}{ccccccccc}
\hline Cell line & Method & \multicolumn{1}{c}{$K_{1}$} & $K_{1} / k_{2}$ & $k_{3}$ & $k_{4}$ & $V_{\mathrm{b}}$ & $D V_{\mathrm{m}}$ & $K_{\mathrm{FLT}}$ \\
\hline A431 $(n=7)$ & 3C5P & $0.227(24.6)$ & $1.860(51.5)$ & $0.123(32.4)$ & $0.011(27.6)$ & $0.033(58.5)$ & $20.64(46.6)$ & $0.103(14.2)$ \\
& 3C4P & $0.163(17.0)$ & $6.349(35.2)$ & $0.021(38.7)$ & - & $0.055(60.2)$ & - & $0.068(18.1)$ \\
& 2C3P & $0.134(14.4)$ & $17.52(34.7)$ & - & - & $0.087(57.7)$ & - & - \\
LLC $(n=10)$ & GPGA & - & - & - & - & - & - & $0.076(15.6)$ \\
& 3C5P & $0.169(26.9)$ & $0.656(14.2)$ & $0.017(29.3)$ & $0.015(22.1)$ & $0.044(45.6)$ & $0.793(39.9)$ & $0.010(20.3)$ \\
& 3C4P & $0.133(28.5)$ & $0.788(9.4)$ & $0.006(30.3)$ & - & $0.059(39.0)$ & - & $0.005(34.2)$ \\
& 2C3P & $0.063(24.5)$ & $1.156(17.7)$ & - & - & $0.093(15.9)$ & - & - \\
& GPGA & - & - & - & - & - & - & $0.003(34.9)$
\end{tabular}

$3 \mathrm{C} 5 \mathrm{P}=3$ compartments and 5 parameters $\left(K_{1}, K_{1} / k_{2}, k_{3}, k_{4}\right.$, and $\left.V_{\mathrm{b}}\right) ; 3 \mathrm{C} 4 \mathrm{P}=3$ compartments and 4 parameters $\left(K_{1}, K_{1} / k_{2}, k_{3}\right.$, and $\left.V_{\mathrm{b}}\right)$, with $k_{4}$ fixed at zero; $2 \mathrm{C} 3 \mathrm{P}=2$ compartments and 3 parameters $\left(K_{1}, K_{1} / k_{2}\right.$, and $\left.V_{\mathrm{b}}\right)$; GPGA $=$ Gjedde-Patlak graphic analysis.

Data are presented as means (CVs). 
TABLE 2

Comparison of Kinetic Models for ${ }^{18} \mathrm{~F}-\mathrm{FLT}$ PET in Mice Implanted with A431 and LLC Tumors by Use of AIC and Extra Sum-of-Squares $F$ Test

\begin{tabular}{|c|c|c|c|c|c|c|}
\hline \multirow[b]{2}{*}{ Cell line } & \multirow[b]{2}{*}{ Mouse } & \multicolumn{2}{|c|}{$\mathrm{AIC}$} & \multirow[b]{2}{*}{ Change in AIC $(1-2)$} & \multirow[b]{2}{*}{$F$ ratio (1 vs. 2 ) } & \multirow[b]{2}{*}{$P$} \\
\hline & & $3 \mathrm{C5P}(1)$ & $3 \mathrm{C} 4 \mathrm{P}(2)$ & & & \\
\hline \multirow[t]{7}{*}{ A431 } & 1 & 354.9 & 389.4 & -34.5 & 2.52 & 0.005 \\
\hline & 2 & 316.5 & 364.0 & -47.4 & 3.58 & $<0.001$ \\
\hline & 3 & 399.4 & 402.2 & -2.8 & 1.07 & 0.424 \\
\hline & 4 & 345.0 & 370.9 & -25.9 & 2.00 & 0.027 \\
\hline & 5 & 340.0 & 376.2 & -36.2 & 2.64 & 0.004 \\
\hline & 6 & 303.9 & 323.5 & -19.6 & 1.69 & 0.071 \\
\hline & 7 & 320.0 & 368.3 & -48.3 & 3.66 & $<0.001$ \\
\hline \multirow[t]{10}{*}{ LLC } & 1 & 322.1 & 371.7 & -49.6 & 3.79 & $<0.001$ \\
\hline & 2 & 302.5 & 331.9 & -29.4 & 2.20 & 0.014 \\
\hline & 3 & 329.7 & 369.7 & -40.0 & 2.92 & 0.002 \\
\hline & 4 & 318.3 & 361.0 & -42.6 & 3.14 & 0.001 \\
\hline & 5 & 319.2 & 351.5 & -32.3 & 2.38 & 0.008 \\
\hline & 6 & 328.7 & 348.4 & -19.7 & 1.69 & 0.070 \\
\hline & 7 & 317.0 & 353.0 & -36.0 & 2.63 & 0.004 \\
\hline & 8 & 333.6 & 382.8 & -49.2 & 3.76 & $<0.001$ \\
\hline & 9 & 325.5 & 363.1 & -37.6 & 2.74 & 0.003 \\
\hline & 10 & 334.3 & 355.9 & -21.6 & 1.78 & 0.053 \\
\hline
\end{tabular}

percentages of intracellular metabolites of ${ }^{18} \mathrm{~F}-\mathrm{FLT}(90.3 \% \pm$ $2.8 \%$ for $\mathrm{A} 431$ and $65.5 \% \pm 8.7 \%$ for LLC) relative to total metabolites and dephosphorylated ${ }^{18} \mathrm{~F}$-FLT, as determined by compartmental analysis.

The correlation coefficients for the ${ }^{18}$ F-FLT PET parameters obtained with the 3C5P model and $120 \mathrm{~min}$ of data and $\%$ Ki-67 are summarized in Table $4 . K_{\mathrm{FLT}}$ (Fig. 7) and $k_{3}$ were found to be highly correlated with $\% \mathrm{Ki}-67(\rho>0.8)$. Furthermore, the distribution volume ratio $\left(k_{3} / k_{4}\right)$ between ${ }^{18}$ F-FLT phosphorylated nucleotides and exchangeable tissue compartment, $D V_{\mathrm{m}}, D V_{\text {tot }}$, and SUV were also highly correlated $(\rho>0.6)$ with $\% \mathrm{Ki}-67$. However, $K_{1}$ and $K_{1} / k_{2}$ were relatively poorly correlated with $\% \mathrm{Ki}-67$.

\section{Feasibility of Simple Models}

We compared $K_{\text {FLT }}$ values obtained with the 3C5P model and PET data obtained over 60, 90, and $120 \mathrm{~min}$ from the initiation of scanning. For A431 tumors, which showed a sufficiently wide $K_{\text {FLT }}$ range for correlation analysis, high correlations were found for $K_{\text {FLT }}$ values obtained from 120min and shorter (60- or 90-min) scans by nonlinear regression analysis (3C5P and 3C4P models) (Table 5). However, undesirable negative correlations were obtained for 60- and 120-min scans for LLC tumors (Table 5). GPGA provided a simple estimate of $K_{\text {FLT }}$ without complex nonlinear curve fitting, but it had estimation bias when compared with the 3C5P model, mainly because of the assumption of irreversible ${ }^{18}$ F-FLT metabolism (Table 1 ). Although $K_{\text {FLT }}$ values estimated with GPGA and 60- or 90-min data showed a significant correlation with $K_{\text {FLT }}$ values obtained with the 120-min 3C5P model for LLC tumors, this correlation was insignificant for A431 tumors (Table 5). SUVs at 80-90 min and 50-60 min showed lower correlations with $K_{\mathrm{FLT}}$ values obtained with the 120-min 3C5P model for both tumor types.
$K_{\mathrm{FLT}}$ values obtained with the 3C5P model and 60 - or 90 min data were consistently higher than 120-min estimates for both cell lines (Table 6 ). $K_{\text {FLT }}$ values obtained with the 90-min 3C5P model for A431 tumors showed a less than $5 \%$ mean difference from those obtained with the 120-min 3C5P model. $K_{\text {FLT }}$ values obtained with 3C4P and GPGA and 60 - or $90-\mathrm{min}$ data were underestimated, although the 3C4P model showed a high correlation for both tumor types (Tables 5 and 6). The correlations between the $K_{\text {FLT }}$ values obtained with the different models and \% Ki-67 are shown in Table 7 . $K_{\text {FLT }}$ values obtained with 60 - or 90 -min data showed a high correlation with $\% \mathrm{Ki}-67$, as did $K_{\text {FLT }}$ values obtained with the 3C5P model and 120-min data (Table 4).

\section{DISCUSSION}

In the present study, we evaluated the validity of an image-derived LV input function in mouse ${ }^{18} \mathrm{~F}$-FLT PET and investigated tracer kinetic models of ${ }^{18}$ F-FLT in mice bearing A431 and LLC tumors. The study revealed that the image-derived arterial input function is feasible for ${ }^{18} \mathrm{~F}$-FLT PET kinetic modeling studies in mice with a simple partialvolume correction and that the 3-compartment model with reversible phosphorylation is most suitable for characterizing the kinetics of ${ }^{18} \mathrm{~F}$-FLT in mice. High correlations of $K_{\mathrm{FLT}}$ and $k_{3}$ with $\% \mathrm{Ki}-67$ were found, a result supporting the notion that these ${ }^{18} \mathrm{~F}$-FLT kinetic parameters in mouse tumor models estimate tumor proliferation.

\section{Input Function}

Quantitative analysis of dynamic PET images with tracer kinetic modeling requires an arterial plasma input function. However, because of small blood vessel diameters and total blood volumes, serial sampling of arterial blood to measure blood activity in mice is difficult (15). Several methods have 


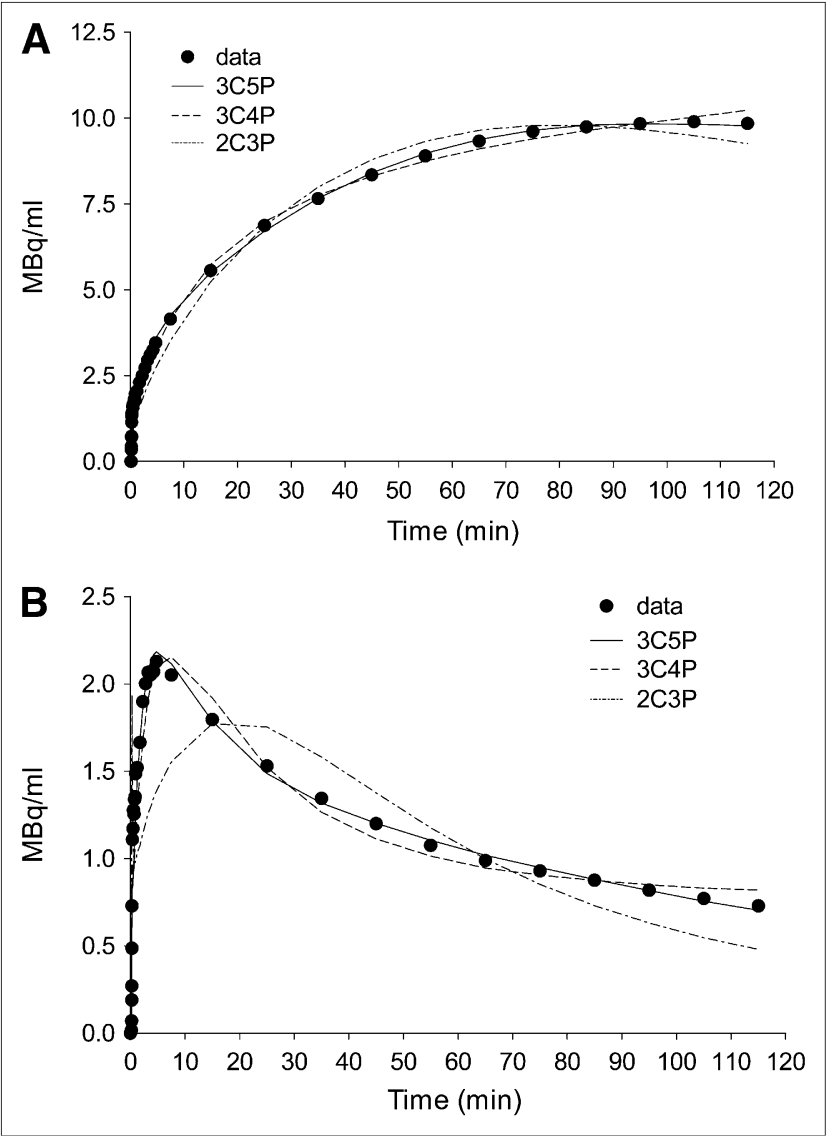

FIGURE 5. Quality of curve fitting obtained with different models and 120 min of data for A431 (A) and LLC (B) tumors. Regression curves defined by 3C5P model were close to data points and did not deviate systematically from data.

been proposed to measure input functions in mice (24-27). Automated blood-sampling devices dedicated to smallanimal imaging can be used (24), but these techniques are limited for multiple-tracer or repetitive studies in the same animals. Image-derived input functions for mouse left ventricles are an alternative to direct blood sampling. This method is attractive, especially in longitudinal studies,

TABLE 3

Average Correlation Coefficients for ${ }^{18}$ F-FLT Kinetic Parameters in Mice Implanted with A431 and LLC Tumors

\begin{tabular}{llrrrrr}
\hline Cell line & Parameter & \multicolumn{1}{c}{$V_{\mathrm{b}}$} & $K_{1}$ & $K_{1} / k_{2}$ & $k_{3}$ & $k_{4}$ \\
\hline A431 & $V_{\mathrm{b}}$ & 1 & - & - & - & - \\
& $K_{1}$ & -0.39 & 1 & - & - & - \\
& $K_{1} / k_{2}$ & 0.29 & -0.79 & 1 & - & - \\
& $k_{3}$ & -0.20 & 0.67 & -0.98 & 1 & - \\
& $k_{4}$ & 0.10 & -0.02 & -0.41 & 0.58 & 1 \\
LLC & $V_{\mathrm{b}}$ & 1 & - & - & - & - \\
& $K_{1}$ & -0.48 & 1 & - & - & - \\
& $K_{1} / k_{2}$ & 0.01 & -0.19 & 1 & - & - \\
& $k_{3}$ & 0.01 & 0.16 & -0.95 & 1 & - \\
& $k_{4}$ & -0.03 & 0.13 & -0.83 & 0.96 & 1 \\
\hline
\end{tabular}
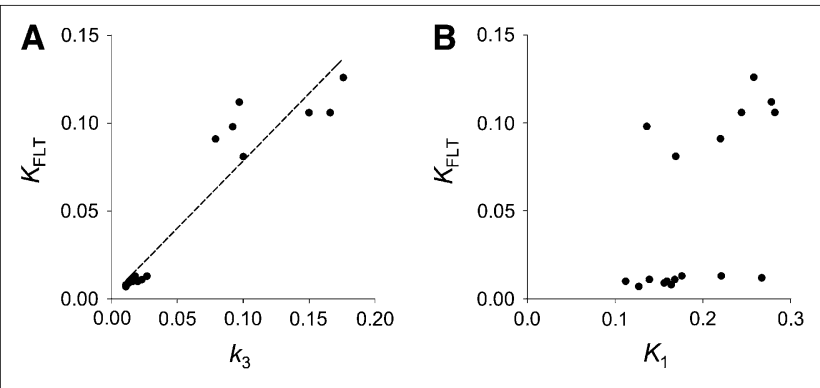

FIGURE 6. Relationships between $k_{3}$ and $K_{\mathrm{FLT}}(r=0.95, P<$ $0.0001)(\mathrm{A})$ and $K_{1}$ and $K_{\mathrm{FLT}}(\mathrm{B})$ for both cell lines ( $\left.n=17\right) . K_{\mathrm{FLT}}$ was better correlated with $k_{3}$ than with $K_{1}$ (flow-dependent parameter).

because of its noninvasive nature. Moreover, considerable improvements in the spatial resolution and sensitivity of state-of-the-art animal PET systems can increase the accuracy of such measurements.

Therefore, we evaluated the validity of image-derived input functions for mouse left ventricles. Rapid equilibration of ${ }^{18} \mathrm{~F}$-FLT between plasma and whole blood was observed at $30 \mathrm{~s}$ after injection, a result indicating that simple imagederived whole-blood activities from left ventricles can be used. In addition, no ${ }^{18} \mathrm{~F}$-FLT metabolite was observed until 2 $\mathrm{h}$ after ${ }^{18} \mathrm{~F}$-FLT injection. The observed low levels of ${ }^{18} \mathrm{~F}$-FLT metabolites in mouse plasma agree with the findings of a previous study (3) and are probably explained by the relatively low uptake and glucuronidation of ${ }^{18} \mathrm{~F}-\mathrm{FLT}$ in mouse liver $(3,18,28)$.

In the present study, a high correlation between arterial blood sample data and PET data was observed, as shown in Figure 3, and the CVs of the ratios of PET data to blood sample data were less than $15 \%$. Actual intersubject variations of this ratio can be expected to be small because blood sampling and PET measurement errors also contribute to these variations. Therefore, these blood activity analysis results suggest that image-derived LV input functions obtained with a state-of-the-art high-resolution rodent PET

TABLE 4

Correlations Between ${ }^{18} \mathrm{~F}$-FLT PET Parameters Obtained from 3C5P Model and 120 Minutes of Data and \% Ki-67

\begin{tabular}{lc}
\hline Parameter & Spearman $\rho$ for $\%$ Ki-67 \\
\hline$K_{1}$ & 0.47 \\
$K_{1} / k_{2}$ & $0.59^{\star}$ \\
$k_{3}$ & $0.86^{\dagger}$ \\
$k_{4}$ & 0.40 \\
$K_{3} / k_{4}$ & $0.77^{\dagger}$ \\
$K_{\mathrm{FLT}}$ & $0.81^{\dagger}$ \\
$D V_{\mathrm{m}}$ & $0.74^{\dagger}$ \\
$D V_{\text {tot }}$ & $0.68^{\dagger}$ \\
$\mathrm{SUV}$ & $0.62^{\dagger}$ \\
& \\
\hline${ }^{*} P<0.05$. & \\
${ }^{\dagger} P<0.01$. & \\
\hline
\end{tabular}




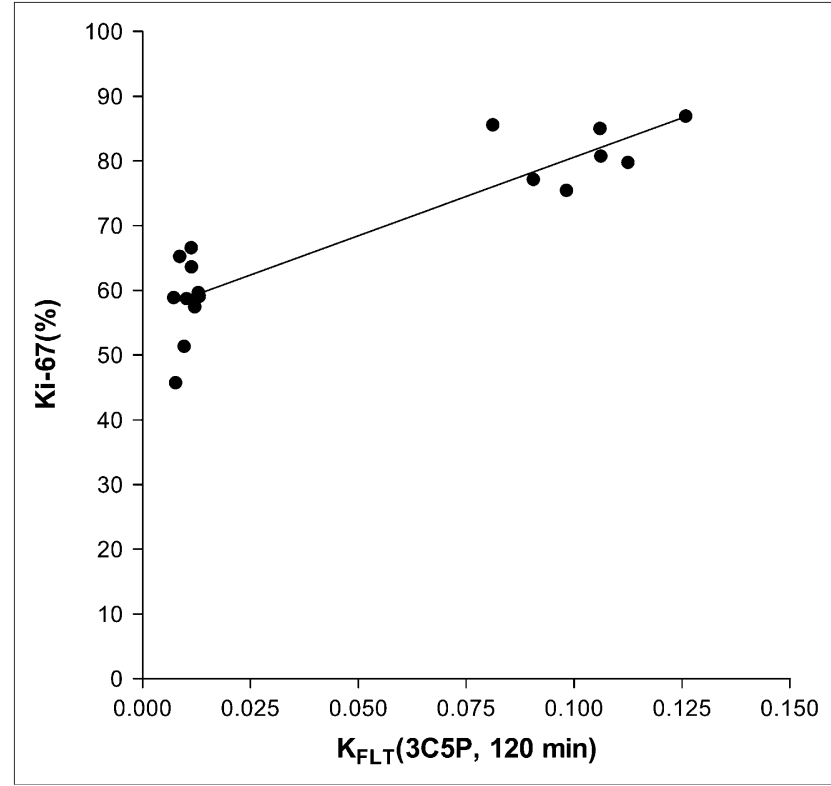

FIGURE 7. Relationship between $K_{\mathrm{FLT}}$ values obtained from $3 \mathrm{C} 5 \mathrm{P}$ model and $120 \mathrm{~min}$ of data and \% Ki-67.

scanner can be used for kinetic modeling of ${ }^{18} \mathrm{~F}$-FLT metabolism in mice.

For investigators who plan to use animals of different sizes or different scanners, a correction factor different from that used in the present study should be obtained because the correction factor is dependent on the spatial resolution of the scanner and the LV size of the animal. If ${ }^{18} \mathrm{~F}$-FLT PET is used for treatment monitoring, then change over time is a primary concern, and such a correction factor can be ignored for simplicity.

\section{Compartmental Modeling}

In human PET data, phosphorylated ${ }^{18}$ F-FLT dephosphorylates more slowly than TK1 activity (29). As shown in Table 1 , dephosphorylation rates $\left(k_{4}\right)$ were also low in the present study and were similar for the 2 tumor cell lines. However, the phosphorylation rate $\left(k_{3}\right)$ in LLC cells was not as rapid as

TABLE 5

Correlations Among ${ }^{18} \mathrm{~F}$-FLT Parameter Estimates Obtained from Different Models

\begin{tabular}{|c|c|c|}
\hline \multirow[b]{2}{*}{ Method } & \multicolumn{2}{|c|}{$\begin{array}{c}\text { Spearman } \rho \text { for } K_{\mathrm{FLT}}, 3 \mathrm{C} 5 \mathrm{P}, \\
120 \mathrm{~min}\end{array}$} \\
\hline & $\mathrm{A} 431(n=7)$ & $\operatorname{LLC}(n=10)$ \\
\hline$K_{\mathrm{FLT}}, 3 \mathrm{C} 5 \mathrm{P}, 90 \mathrm{~min}$ & $0.98^{*}$ & $0.82^{*}$ \\
\hline$K_{\mathrm{FLT}}, 3 \mathrm{C} 5 \mathrm{P}, 60 \mathrm{~min}$ & $1.00^{*}$ & $-0.69^{\dagger}$ \\
\hline$K_{\mathrm{FLT}}, 3 \mathrm{C} 4 \mathrm{P}, 60 \mathrm{~min}$ & $0.88^{*}$ & $0.91^{*}$ \\
\hline$K_{\mathrm{FLT}}, \mathrm{GPGA}, 90 \mathrm{~min}$ & 0.39 & $0.90^{\star}$ \\
\hline$K_{\mathrm{FLT}}, \mathrm{GPGA}, 60 \mathrm{~min}$ & 0.56 & $0.83^{\star}$ \\
\hline SUV, $80-90 \mathrm{~min}$ & 0.39 & -0.09 \\
\hline SUV, $50-60 \mathrm{~min}$ & 0.39 & -0.29 \\
\hline $\begin{array}{l}{ }^{\star} P<0.01 \\
{ }^{\dagger} P<0.05\end{array}$ & & \\
\hline
\end{tabular}

TABLE 6

Average Percentage Changes in ${ }^{18} \mathrm{~F}-\mathrm{FLT}$ Net Influx

Parameters $\left(K_{\mathrm{FLT}}\right)$ Obtained from 3C5P Model and 120 Minutes of Data

\begin{tabular}{ccc}
\hline & \multicolumn{2}{c}{ Mean \pm SD \% change } \\
\cline { 2 - 3 } Method & A431 $(n=7)$ & LLC $(n=10)$ \\
\hline 3C5P, 90 min & $15.2 \pm 3.4$ & $27 \pm{ }^{18} .1$ \\
3C5P, 60 min & $-18 \pm 6.3$ & $100.5 \pm 64.3$ \\
3C4P, 60 min & $-26.1 \pm 9.8$ & $-63.4 \pm 6.8$ \\
GPGA, 90 min & $-18.6 \pm 7.6$ & $-56.6 \pm 8.3$ \\
GPGA, 60 min &
\end{tabular}

that in A431 cells and was similar to the dephosphorylation rate. Thus, LLC had a low level of ${ }^{18}$ F-FLT retention, as shown in Figure 4 . This finding suggests that $k_{4}$ should not be ignored in the kinetic modeling of the LLC cell line when 120 min of data are used. $K_{\mathrm{FLT}}$, which was calculated on the basis of the assumption that $k_{4}$ can be ignored, was underestimated by $69 \%$ in this cell line (Table 1). A significant loss of phosphorylated ${ }^{18}$ F-FLT nucleotides was also evident in kinetic studies of non-small cell lung cancer (11) and gliomas (12). However, previous ${ }^{18} \mathrm{~F}$-FLT kinetic studies in dogs bearing non-Hodgkin's lymphoma and soft-tissue sarcoma (30) and in patients with colorectal cancer (9) demonstrated that the incorporation of $k_{4} \neq 0$ is not necessary to improve model accuracy. In these studies, dynamic PET scans were acquired for only up to $60 \mathrm{~min}$, a length of time that might not have been sufficient to accurately estimate the effects of the $k_{4}$ parameter. Further systemic investigation of the issue of $k_{4} \neq 0$ in ${ }^{18} \mathrm{~F}$-FLT kinetics is necessary because there are nonbiochemical factors that can lead to better curve fitting with $k_{4} \neq 0$. Heterogeneity of tissue composition is an example of such a factor.

In the present study, all compartment models had lower AIC values when the blood volume fraction term was included; this finding means that this parameter should not be neglected in ${ }^{18}$ F-FLT kinetic models. Blood volume fractions also showed wide variations in both tumor cell lines (Table 1), a result that may have been attributable, in part, to variable vasculature changes in tumors. In addition, tumor-bearing regions showed higher blood volume fractions than did

\section{TABLE 7}

Correlations Between $K_{\mathrm{FLT}}$ Values Obtained from Different Models and \% $\mathrm{Ki}-67$

\begin{tabular}{cc}
\hline Method & Spearman $\rho$ for $\% \mathrm{Ki}-67^{\star}$ \\
\hline 3C5P, $90 \mathrm{~min}$ & 0.77 \\
3C5P, $60 \mathrm{~min}$ & 0.76 \\
3C4P, $60 \mathrm{~min}$ & 0.80 \\
GPGA, $90 \mathrm{~min}$ & 0.72 \\
GPGA, $60 \mathrm{~min}$ & 0.73 \\
\hline${ }^{*} P<0.01$ for all values. \\
\hline
\end{tabular}


normal regions, a result that is probably associated with enhanced neovascularization in these tumor models (31). With regard to the use of ${ }^{18} \mathrm{~F}$-FLT PET for the assessment of therapeutic outcomes, considerations of blood volume fractions are important because of the possible effects of increased edema induced by treatment (13).

\section{Model Parameters}

$K_{\mathrm{FLT}}$ and $k_{3}$ showed high correlations with $\% \mathrm{Ki}-67$, but no correlation of $K_{1}$ and $k_{4}$ with $\%$ Ki-67 was observed (Table 4). The strong correlation of $K_{\mathrm{FLT}}$ with $\% \mathrm{Ki}-67$ seems to be related to the parameter $k_{3}$, which is directly associated with TK1 activity, because $K_{\mathrm{FLT}}$ is not flow-dependent (Fig. 6) and $K_{1}$ levels were similar between the 2 tumor models. Better correlations between $k_{3}$ or $K_{\mathrm{FLT}}$ and \% Ki-67 than between SUVs and \% Ki-67 may be attributable to a significant amount of label loss because of dephosphorylation $\left(k_{4}\right)$ and better normalization of tumoral activity by input function integration rather than use of the injected dose.

In general, macroparameters, that is, combinations of microparameters, were more stable than individual microparameters. Therefore, associations between several macroparameters $\left(k_{3} / k_{4}, K_{\mathrm{FLT}}, D V_{\mathrm{m}}\right.$, and $\left.D V_{\mathrm{tot}}\right)$ and ${ }^{18} \mathrm{~F}-\mathrm{FLT}$ metabolism were explored in the present study. Although all were found to be useful for differentiating A431 tumors and LLC tumors, $K_{\mathrm{FLT}}$ values in tumor-bearing regions had the smallest variability, as shown in Table 1 . In addition, $K_{\mathrm{FLT}}$ showed a strong correlation with $k_{3}$ (Fig. 6).

\section{Simple Models}

Our results show that PET parameters $\left(k_{3}\right.$ and $\left.K_{\text {FLT }}\right)$ estimated with the 3C5P model and 120 min of PET data reflect well the metabolism of ${ }^{18} \mathrm{~F}$-FLT in tumor cells. However, PET of mice for $120 \mathrm{~min}$ is inconvenient because of the time involved and the possibility of animal movement. The possible biologic effects of long-term anesthesia are also problematic.

Unfortunately, $K_{\mathrm{FLT}}$ estimates based on the 60 - or 90 -min 3C5P model were consistently higher than $K_{\mathrm{FLT}}$ estimates based on the 120-min model, and those obtained from the 3C4P model were lower. Only in tumor models with high uptake (A431 models) did 90-min $K_{\text {FLT }}$ estimates differ from 120 -min estimates by less than $5 \%$. This result suggests that the estimation of $k_{4}$ is a vital element and that 60 -min data are inappropriate in these tumor models if the absolute quantification of $K_{\text {FLT }}$ is important.

If the specific aim of an ${ }^{18} \mathrm{~F}$-FLT PET study is to monitor the therapeutic response after cancer treatment, then assessment of relative changes in a kinetic parameter over time will be sufficient. Despite the overestimation of $K_{\mathrm{FLT}}$ values, the 60- or 90-min 3C5P model had a strong correlation with the 120-min model in highly proliferative tumor cells (A431). Although $K_{\text {FLT }}$ values obtained with the 60-min 3C5P model in LLC tumors had a negative correlation with those obtained with the 120 -min 3C5P model and showed a high relative percentage of change, these results were mainly attributable to the fact that LLC tumors have a very low level and narrow range of $K_{\mathrm{FLT}}$ values; these conditions are not appropriate for these comparisons. In addition, $K_{\text {FLT }}$ values obtained with shorter scan times (60 or $90 \mathrm{~min}$ ) showed a high correlation with \% Ki-67 (Table 7), as did $K_{\text {FLT }}$ values obtained with the 3C5P model and $120 \mathrm{~min}$ of data. Although there is still controversy regarding the appropriateness of the incorporation of $k_{4}$ in an ${ }^{18} \mathrm{~F}$-FLT kinetic analysis with $60 \mathrm{~min}$ of data, these experimental results support the notion that a dynamic ${ }^{18}$ F-FLT PET scan time shorter than 120 min can be used if the only parameter of interest is the relative change in $K_{\text {FLT }}$. In addition, the incorporation of $k_{4}$ in a kinetic analysis of shorter scan data seems to be feasible; this factor is important in the accurate estimation of $K_{\mathrm{FLT}}$ in therapeutic response monitoring with possibly varying $k_{4}$ values $(11)$.

$K_{\mathrm{FLT}}$ estimates based on the 3C4P model $\left(k_{4}=0\right)$ and 60 min of data correlated well with those based on the full model and data (3C5P and $120 \mathrm{~min}$ ), as shown in Table 5, and the level of underestimation of $K_{\text {FLT }}$ was not significant. Therefore, this model will also be useful in some applications in which only the relative change in $K_{\text {FLT }}$ is important and in which $K_{\mathrm{FLT}}$ is insensitive to changes in $k_{4}$ or the assumption of no changes in $k_{4}$ can be justified.

\section{Limitations}

This study has several limitations. First, we used a relatively small number of mice for each tumor model. Nevertheless, consistent results were obtained with respect to model selection and parameter estimation. Second, concentrations of serum thymidine and the lumped constant for ${ }^{18} \mathrm{~F}$ FLT were not considered. Although the consideration of a lumped constant, which accounts for differences in the transport and phosphorylation of ${ }^{18} \mathrm{~F}$-FLT and thymidine, is required, no systematic study of this issue has been undertaken (32). Finally, 30-46 MBq of ${ }^{18}$ F-FLT were injected into mice to analyze ${ }^{18} \mathrm{~F}$-FLT metabolites more accurately in tumors, because initial studies with $7.5 \mathrm{MBq}$ of ${ }^{18} \mathrm{~F}$-FLT did not result in a peak in LLC tumors. However, the effects of a perturbation of cellular proliferation on study results are likely to be negligible.

\section{CONCLUSION}

The present study showed that the image-derived arterial input function is feasible for kinetic modeling studies of ${ }^{18}$ F-FLT PET in mice. For 120-min dynamic PET data, the 3-compartment model with reversible phosphorylation and a variable blood volume fraction was found to best describe tissue time-activity curves in tumor-bearing regions. $K_{\mathrm{FLT}}$ values obtained with this model showed reasonable intersubject variability and discrimination ability for tumor models with different proliferation properties. At least 90 min of data are necessary to obtain accurate absolute $K_{\mathrm{FLT}}$ values for $\mathrm{A} 431$ with the $3 \mathrm{C} 5 \mathrm{P}$ model. Our results also suggested that imaging for $60 \mathrm{~min}$ is useful in some applications, such as cancer treatment monitoring, in which the 
relative change in the $K_{\mathrm{FLT}}$ parameter over time is more important than absolute values.

\section{ACKNOWLEDGMENTS}

This work was supported by the Real-Time Molecular Imaging Research Program and Basic Research Program (R01-2006-000-10296-0) of the Korean Science \& Engineering Foundation, the Korea Health 21 R\&D Project, the Ministry of Health \& Welfare (grant A062254), and the Seoul R\&BD Program (grant 10550) managed by the Seoul Development Institute. The authors would like to acknowledge that this study was assisted by the support of the GigaNetwork (KREONET) of KISTI.

\section{REFERENCES}

1. Sherley JL, Kelly TJ. Regulation of human thymidine kinase during the cell cycle. J Biol Chem. 1988;263:8350-8358.

2. Grierson JR, Shields AF. Radiosynthesis of $3^{\prime}$-deoxy- $3^{\prime}-\left[{ }^{18} \mathrm{~F}\right]$ fluorothymidine: $\left[{ }^{18} \mathrm{~F}\right] \mathrm{FLT}$ for imaging of cellular proliferation in vivo. $\mathrm{Nucl} \mathrm{Med}$ Biol. 2000;27:143-156.

3. Barthel H, Cleij MC, Collingridge DR, et al. $3^{\prime}-$ Deoxy- $3^{\prime}-\left[{ }^{18} \mathrm{~F}\right]$ fluorothymidine as a new marker for monitoring tumor response to antiproliferative therapy in vivo with positron emission tomography. Cancer Res. 2003;63:3791-3798.

4. Wagner M, Seitz U, Buck A, et al. $3^{\prime}-\left[{ }^{18} \mathrm{~F}\right]$ Fluoro-3'-deoxythymidine ( $\left[{ }^{18} \mathrm{~F}\right]$ FLT) as positron emission tomography tracer for imaging proliferation in a murine B-cell lymphoma model and in the human disease. Cancer Res. 2003;63: 2681-2687.

5. Choi SJ, Kim JS, Kim JH, et al. $\left[{ }^{18} \mathrm{~F}\right] 3^{\prime}$-Deoxy-3'-fluorothymidine PET for the diagnosis and grading of brain tumors. Eur J Nucl Med Mol Imaging. 2005;32: 653-659.

6. Kenny LM, Vigushin DM, Al-Nahhas A, et al. Quantification of cellular proliferation in tumor and normal tissues of patients with breast cancer by $\left[{ }^{18} \mathrm{~F}\right]$ fluorothymidine-positron emission tomography imaging: evaluation of analytical methods. Cancer Res. 2005;65:10104-10112.

7. Waldherr C, Mellinghoff IK, Tran C, et al. Monitoring antiproliferative responses to kinase inhibitor therapy in mice with $3^{\prime}$-deoxy- $3^{\prime}-{ }^{18} \mathrm{~F}$-fluorothymidine PET. J Nucl Med. 2005;46:114-120.

8. Muzi M, Mankoff DA, Grierson JR, Wells JM, Vesselle H, Krohn KA. Kinetic modeling of $3^{\prime}$-deoxy-3'-fluorothymidine in somatic tumors: mathematical studies. J Nucl Med. 2005;46:371-380.

9. Visvikis D, Francis D, Mulligan R, et al. Comparison of methodologies for the in vivo assessment of 18FLT utilisation in colorectal cancer. Eur J Nucl Med Mol Imaging. 2004;31:169-178.

10. Jacobs AH, Thomas A, Kracht LW, et al. ${ }^{18} \mathrm{~F}$-Fluoro-L-thymidine and ${ }^{11} \mathrm{C}$ methylmethionine as markers of increased transport and proliferation in brain tumors. J Nucl Med. 2005;46:1948-1958.
11. Muzi M, Vesselle H, Grierson JR, et al. Kinetic analysis of $3^{\prime}$-deoxy-3'fluorothymidine PET studies: validation studies in patients with lung cancer. J Nucl Med. 2005;46:274-282.

12. Muzi M, Spence AM, O'Sullivan F, et al. Kinetic analysis of $3^{\prime}$-deoxy- $3^{\prime}-{ }^{18} \mathrm{~F}-$ fluorothymidine in patients with gliomas. J Nucl Med. 2006;47:1612-1621.

13. Schiepers C, Chen W, Dahlbom M, Cloughesy T, Hoh CK, Huang SC. ${ }^{18} \mathrm{~F}-$ fluorothymidine kinetics of malignant brain tumors. Eur J Nucl Med Mol Imaging. 2007;34:1003-1011.

14. Hume SP, Myers R. Dedicated small animal scanners: a new tool for drug development? Curr Pharm Des. 2002;8:1497-1511.

15. Pomper MG, Lee JS. Small animal imaging in drug development. Curr Pharm Des. 2005;11:3247-3272.

16. Kim TJ, Ravoori M, Landen CN, et al. Antitumor and antivascular effects of AVE8062 in ovarian carcinoma. Cancer Res. 2007;67:9337-9345.

17. Lee JS, Orita $\mathrm{H}$, Gabrielson $\mathrm{K}$, et al. FDG-PET for pharmacodynamic assessment of the fatty acid synthase inhibitor C75 in an experimental model of lung cancer. Pharm Res. 2007;24:1202-1207.

18. Sugiyama M, Sakahara H, Sato $\mathrm{K}$, et al. Evaluation of $3^{\prime}$-deoxy- $3^{\prime}-{ }^{18} \mathrm{~F}-$ fluorothymidine for monitoring tumor response to radiotherapy and photodynamic therapy in mice. J Nucl Med. 2004;45:1754-1758.

19. Oyama N, Ponde DE, Dence C, Kim J, Tai YC, Welch MJ. Monitoring of therapy in androgen-dependent prostate tumor model by measuring tumor proliferation. J Nucl Med. 2004;45:519-525.

20. Yang YJ, Ryu JS, Kim SY, et al. Use of $3^{\prime}$-deoxy- $3^{\prime}-\left[{ }^{18} \mathrm{~F}\right]$ fluorothymidine PET to monitor early responses to radiation therapy in murine SCCVII tumors. Eur $J$ Nucl Med Mol Imaging. 2006;33:412-419.

21. Lee SJ, Oh SJ, Chi DY, et al. Simple and highly efficient synthesis of 3 '-deoxy$3^{\prime}-\left[{ }^{18} \mathrm{~F}\right]$ fluorothymidine using nucleophilic fluorination catalyzed by protic solvent. Eur J Nucl Med Mol Imaging. 2007;34:1406-1409.

22. Kim JS, Lee JS, Im KC, et al. Performance measurement of the microPET Focus 120 scanner. J Nucl Med. 2007;48:1527-1535.

23. Burger C, Buck A. Requirements and implementation of a flexible kinetic modeling tool. J Nucl Med. 1997;38:1818-1823.

24. Wu HM, Sui G, Lee CC, et al. In vivo quantitation of glucose metabolism in mice using small-animal PET and a microfluidic device. J Nucl Med. 2007;48:837-845.

25. Ferl GZ, Zhang X, Wu HM, Huang SC. Estimation of the ${ }^{18} \mathrm{~F}-\mathrm{FDG}$ input function in mice by use of dynamic small-animal PET and minimal blood sample data. J Nucl Med. 2007;48:2037-2045.

26. Kim J, Herrero P, Sharp T, et al. Minimally invasive method of determining blood input function from PET images in rodents. J Nucl Med. 2006;47:330-336.

27. Laforest R, Sharp TL, Engelbach JA, et al. Measurement of input functions in rodents: challenges and solutions. Nucl Med Biol. 2005;32:679-685.

28. Nicolas F, De Sousa G, Thomas P, Placidi M, Lorenzon G, Rahmani R. Comparative metabolism of 3'-azido-3'-deoxythymidine in cultured hepatocytes from rats, dogs, monkeys, and humans. Drug Metab Dispos. 1995;23:308-313.

29. Grierson JR, Schwartz JL, Muzi M, Jordan R, Krohn KA. Metabolism of 3'deoxy-3'-[F-18]fluorothymidine in proliferating A549 cells: validations for positron emission tomography. Nucl Med Biol. 2004;31:829-837.

30. Shields AF, Grierson JR, Muzik O, et al. Kinetics of $3^{\prime}$-deoxy-3'-[F- $\left.{ }^{18}\right]$ fluorothymidine uptake and retention in dogs. Mol Imaging Biol. 2002;4:83-89.

31. Myoken Y, Kayada Y, Okamoto T, Kan M, Sato GH, Sato JD. Vascular endothelial cell growth factor (VEGF) produced by A-431 human epidermoid carcinoma cells and identification of VEGF membrane binding sites. Proc Natl Acad Sci USA. 1991;88:5819-5823.

32. Barthel H, Aboagye E, Price P. Author reply. Cancer Res. 2003;63:8560. 